\title{
On Quantum Systems in Thermal Contact
}

\author{
H. RoOS \\ Institut für Theoretische Physik der Universität Göttingen
}

Received August 27, 1971; in revised form February 21, 1972

\begin{abstract}
It is shown that under rather general conditions two K.M.S. states $\omega_{1}$ and $\omega_{2}$ of systems $S_{1}$ and $S_{2}$, respectively, can be simultaneously extended to a K.M.S. state $\omega$ of a system composed of $S_{1}$ and $S_{2}$, provided both systems have equal temperatures. This result gives further support to the conjecture that K.M.S. states are equilibrium states. In the second part, a model of thermal coupling is constructed which satisfies the assumptions of the first part, thereby showing that the result is also valid in the interesting case of systems $S_{1}$ and $S_{2}$ in thermal contact.
\end{abstract}

\section{Introduction}

During the past three years, there appeared an increasing number of papers on the Kubo-Martin-Schwinger boundary condition strongly supporting the assumption that a state $\omega$ of a general quantum statistical system is an equilibrium state if and only if $\omega$ satisfies the K.M.S. condition [1-6]. It is understood that the systems under consideration do not contain adiabatically closed subsystems of different temperatures. There are proofs of this conjecture for special cases [1-4], but, to my knowledge, no general proof exists. This paper is intended to give further support to the above conjecture.

If we consider two systems $S_{1}$ and $S_{2}$ described by the respective algebras of observables $\mathfrak{H}_{1}, \mathfrak{I}_{2}$ and the states $\omega_{1}$ and $\omega_{2}$ which satisfy the K.M.S. condition, we may ask whether $\omega_{1}$ and $\omega_{2}$ have a common extension to a K.M.S. state $\omega$ of a system composed by $S_{1}$ and $S_{2}$, provided $S_{1}$ and $S_{2}$ have equal temperatures. If K.M.S. states are equilibrium states, we expect this to be true. In the next section, we shall give a proof of this conjecture using rather general assumptions. In Section III, we shall exhibit a model of thermal coupling of two finite quantum systems. Finally, we shall deal with the infinite volume limit and show that the systems are continuous in the coupling parameter, and, in addition, we shall argue that the states of the infinite systems are essentially the same as those of the uncoupled systems if the temperatures are equal. The results of Sections III and IV are intended to show that the assumptions of Section II are reasonable even in the presence of thermal coupling. 


\section{Simultaneous Extension of KMS States}

Let $\mathfrak{A}$ be a $C^{*}$-algebra, $\alpha_{t} \in$ Aut $\mathfrak{A}$ a representation of the group of time translations as automorphisms of $\mathfrak{A}$. The K.M.S. condition can be stated in the following form:

Definition 2.1. $\omega$ is a K.M.S. state over $\mathfrak{A}$ with respect to $\alpha_{t}$ belonging to the inverse temperature $\beta=\frac{1}{k T}$, if for all $A, B \in \mathfrak{A}$

(i) $\omega\left(A \alpha_{t} B\right)$ is a continuous function of $t$,

(ii) $\int f(t-i \beta) \omega\left(A \alpha_{t} B\right) d t=\int f(t) \omega\left(\left(\alpha_{t} B\right) A\right) d t$ for all $f(t)$ with Fourier transform $\mathscr{F} f \in \mathscr{D}$, where $\mathscr{D}$ denotes the set of
infinitely differentiable functions with compact support.

Define the algebra of holomorphic elements of $\mathfrak{A}$ by

Definition 2.2. $\hat{\mathfrak{U}}=\left\{A \in \mathfrak{A} ; \alpha_{t} A\right.$ is a holomorphic operator-valued function of $t\}$.

$\hat{\mathfrak{Q}}$ is not empty; if, for instance, $\mathscr{F} f \in \mathscr{D}$, then $\int f(t) \alpha_{t} A d t \in \hat{\mathfrak{A}}$. If we restrict ourselves to elements of $\hat{\mathfrak{A}}$, there is another formulation of the condition (KMS):

$$
\omega\left(A \alpha_{i \beta} B\right)=\omega(B A), \quad A \in \mathfrak{A}, B \in \hat{\mathfrak{A}} .
$$

$\left(\mathrm{KMS}^{\prime}\right)$

From $\left(\mathrm{KMS}^{\prime}\right)$, we cannot conclude that $\omega$ is a K.M.S. state unless $\hat{\mathfrak{O}}$ is dense in $\mathfrak{A}$. However, for $A \in \mathfrak{A}, B \in \hat{\mathfrak{A}}$, the conditions (KMS) and $\left(\mathrm{KMS}^{\prime}\right)$ are completely equivalent [7]. Norm-density of $\hat{\mathfrak{A}}$ in $\mathfrak{A}$ is implied by strong continuity of $\alpha_{t}$, i.e. $\left\|\alpha_{t} A-A\right\| \underset{t \rightarrow 0}{\longrightarrow} 0$ for all $A \in \mathfrak{A}$. But there is a result by Takesaki and Winnink [8] stating that in case of a strongly continuous group of time automorphisms on a von Neumann algebra $\mathfrak{A}$ the existence of a K.M.S. state over $\mathfrak{A}$ implies that $\mathfrak{A}$ is of finite type. Therefore, we shall not assume strong continuity of $\alpha_{t}$ and use condition (KMS) to define K.M.S. states. In addition, we shall use the equivalence of (KMS) and (KMS') on the subset $\hat{\mathfrak{A}}$.

We consider two quantum statistical systems. Let $\mathfrak{A}_{1}$ and $\mathfrak{I}_{2}$ be the respective $C^{*}$-algebras of observables. We need the following assumptions:

(A) $\mathfrak{U}_{1}$ and $\mathfrak{I}_{2}$ are embedded in a larger $C^{*}$-algebra $\mathfrak{N}_{0}$ so that we can define $\mathfrak{N}_{12}$ as the $C^{*}$-algebra generated by $\mathfrak{I}_{1}$ and $\mathfrak{I}_{2}$.

(B) $\mathfrak{A}_{1}$ and $\mathfrak{A}_{2}$ commute elementwise.

(C) For any $A \in \mathfrak{A}_{1}$, and any $B \in \mathfrak{Q}_{2}$ we have $A B \neq 0$ whenever $A \neq 0$, $B \neq 0$.

These assumptions seem reasonable. Let us consider, for instance, two infinite regions $\Lambda_{1}, \Lambda_{2}$ with $\Lambda_{1} \cup \Lambda_{2}=\mathbb{R}^{3}, \Lambda_{1} \cap \Lambda_{2}=\emptyset$.

Let $L_{i}, i=1,2$, denote two Hilbert spaces of functions with supp $f_{i} \subset \Lambda_{i}$, $f_{i} \in L_{i}$, and let $\mathfrak{I}_{i}$ be the corresponding representations of the $C^{*}$-algebras 
$\tilde{\mathfrak{A}}_{i}, i=1,2$, of the canonical commutation relations (CCR). Then $\mathfrak{A}_{12}$ is the representation of the CCR corresponding to $L=L_{i} \oplus L_{2}$. It is then clear, that (A) and (B) are fulfilled.

Furthermore, for any representation of the CCR we have $[9,10]$

$$
\pi\left(\tilde{\mathfrak{U}}_{1}\right) \cong \pi\left(\tilde{\mathfrak{U}}_{1}\right) \otimes \mathbf{1}, \pi\left(\tilde{\mathfrak{U}}_{2}\right) \cong \mathbf{1} \otimes \pi\left(\tilde{\mathfrak{U}}_{2}\right),
$$

and thus $(\mathrm{C})$ is valid.

To give another example, we may construct, following Ruelle [11], a quasilocal algebra $\mathfrak{U}$, starting with $\mathfrak{U}_{\Lambda}=\mathfrak{B}\left(\mathscr{H}_{A}\right)=$ von Neumannalgebra of all bounded operators on $\mathscr{H}_{A}$. $\mathscr{H}_{A}$ denote Hilbert spaces with $\mathscr{H}_{\Lambda} \cong \mathscr{H}_{\Lambda \backslash \Lambda_{1}} \otimes \mathscr{H}_{\Lambda_{1}}$ whenever $\Lambda_{1} \subset \Lambda$. With $\mathfrak{U}_{i}=\bigcup_{\Lambda \subset \Lambda_{l}} \mathfrak{U}_{\Lambda}, i=1$, , we have

$$
\mathfrak{A}_{12}=\mathfrak{A}=\bar{\bigcup} \mathfrak{A}_{\Lambda}, \quad \mathfrak{A}_{1} \cong \mathfrak{A}_{1} \otimes 1, \quad \mathfrak{A}_{2} \cong 1 \otimes \mathfrak{A}_{2},
$$

and our assumptions are fulfilled. This example refers to the case of bosons; for fermion systems one has to use the "even" algebra $\mathfrak{A}^{e}$ (cf. [11], p. 178).

We have to make two further assumptions:

(D) There exist two groups $\alpha_{t}^{1}, \alpha_{t}^{2}$ of automorphisms of $\mathfrak{I}_{1}$ and $\mathfrak{I}_{2}$, respectively: $\alpha_{t}^{i} \mathfrak{A}_{i} \subset \mathfrak{A}_{i}$, which are representations of the time translations.

We expect this to be true of separated systems. As to systems in thermal contact, we refer to the discussion in the next sections. Our last assumption is a technical one:

(E) The automorphisms $\alpha_{t}^{1}$ and $\alpha_{t}^{2}$ can be extended to automorphisms $\alpha_{t}$ of $\mathfrak{Q}_{12}$.

Assumption (E) will be shortly discussed later. Now let us consider K.M.S. states $\omega_{1}$ and $\omega_{2}$ of our systems and ask whether there exists a K.M.S. state $\omega$ on $\mathfrak{A}_{12}$ whose restrictions to $\mathfrak{A}_{i}$ equal $\omega_{i}, i=1,2$. Regarding K.M.S. states as equilibrium states we expect a positive answer if $\omega_{1}$ and $\omega_{2}$ belong to the same $\beta$.

Indeed, we have the following

Theorem 2.3. Let $\mathfrak{A}_{1}, \mathfrak{I}_{2}$ be $C^{*}$-algebras for which the assumptions $(A)$ to $(E)$ are satisfied, and let $\omega_{1}, \omega_{2}$ be K.M.S. states over $\mathfrak{I}_{1}$ and $\mathfrak{A}_{2}$, respectively, belonging to the inverse temperatures $\beta_{1}$ and $\beta_{2}$. Then there exists a K.M.S. state $\omega$ over $\mathfrak{A}_{12}$ which is a common extension of $\omega_{1}$ and $\omega_{2}$ if and only if $\beta_{1}=\beta_{2}$.

Proof. The "only if" direction is trivial: the restrictions on $\mathfrak{A}_{i}$ of any $\beta$-K.M.S. state $\omega$ are $\beta$-K.M.S. states, too.

In the following, we suppose that $\omega_{1}$ and $\omega_{2}$ are $\beta$-K.M.S. states. It is known [12] that the assumptions (A), (B), (C) imply that $\mathfrak{A}_{1}$ and $\mathfrak{A}_{2}$ are statistically independent, i.e.

11 Commun. math. Phys, Vol. 26 
(i) there exists a homomorphism $\Phi: \mathfrak{A}_{12} \rightarrow \mathfrak{A}_{1} \otimes_{\alpha} \mathfrak{H}_{2}$, where $\mathfrak{U}_{1} \otimes_{\alpha} \mathfrak{H}_{2}$ denotes the closure in the $\alpha$-norm [13] of the direct algebraic product $\mathfrak{A}_{1} \odot \mathfrak{U}_{2}$;

(ii) there exists a common extension of any two states $\omega_{1}, \omega_{2}$ over $\mathfrak{A}_{1}$ and $\mathfrak{U}_{2}$ respectively, to a state $\omega$ over $\mathfrak{A}_{12}$, given by $\omega=\omega_{1} \otimes \omega_{2} \circ \Phi$. We shall show that $\omega$ is a $\beta$-K.M.S. state.

On the set

$$
\mathfrak{U}_{1} \vee \mathfrak{U}_{2}=\left\{\sum_{\text {finite }} A_{i}^{1} A_{i}^{2} ; A_{i}^{1} \in \mathfrak{A}_{1}, A_{i}^{2} \in \mathfrak{U}_{2}\right\},
$$

the norm-closure of which equals $\mathfrak{A}_{12}$, the homomorphism $\Phi$ is actually an isomorphism, given by

$$
\Phi \sum_{\text {finite }} A_{i}^{1} A_{i}^{2}=\sum_{\text {finite }} A_{i}^{1} \otimes A_{i}^{2} \in \mathfrak{U}_{1} \odot \mathfrak{A}_{2} .
$$

On $\mathfrak{A}_{1} \odot \mathfrak{A}_{2}$, we define automorphisms $\alpha_{t}^{1} \odot \alpha_{t}^{2}$ by

$$
\alpha_{t}^{1} \odot \alpha_{t}^{2} \Sigma A_{i}^{1} \otimes A_{i}^{2}=\Sigma\left(\alpha_{t}^{1} A_{i}^{1}\right) \otimes\left(\alpha_{t}^{2} A_{i}^{2}\right) ;
$$

clearly, we have

$$
\Phi \alpha_{t}=\alpha_{t}^{1} \odot \alpha_{t}^{2} \Phi \quad \text { on } \quad \mathfrak{A}_{1} \vee \mathfrak{A}_{2} .
$$

Let us compute the $\alpha$-norm of $\alpha_{t}^{1} \odot \alpha_{t}^{2}$.

$$
\begin{aligned}
& \left\|\alpha_{t}^{1} \odot \alpha_{t}^{2} \sum_{i=1}^{n} A_{i}^{1} \otimes A_{i}^{2}\right\|_{\alpha}=\left\|\sum_{i=1}^{n} \alpha_{t}^{1} A_{i}^{1} \otimes \alpha_{t}^{2} A_{i}^{2}\right\|_{\alpha} \\
& =\sup \left\{\frac{\varphi_{1} \otimes \varphi_{2}\left[\left(\sum_{1}^{m} C_{i} \otimes D_{i}\right)^{*}\left(\sum_{1}^{n} \alpha_{t}^{1} A_{j}^{1} \otimes \alpha_{t}^{2} A_{j}^{2}\right)^{*}\left(\sum_{1}^{n} \alpha_{t}^{1} A_{k}^{1} \otimes \alpha_{t}^{2} A_{k}^{2}\right)\left(\sum_{1}^{m} C_{l} \otimes D_{l}\right)\right]}{\varphi_{1} \otimes \varphi_{2}\left[\left(\sum_{1}^{m} C_{i} \otimes D_{i}\right)^{*}\left(\sum_{1}^{m} C_{l} \otimes D_{l}\right)\right]}\right]^{1 / 2}
\end{aligned}
$$

where the supremum is to be taken over all states $\varphi_{1}$ over $\mathfrak{A}_{1}$, all states $\varphi_{2}$ over $\mathfrak{U}_{2}$, all $C_{j} \in \mathfrak{U}_{1}$ and all $D_{j} \in \mathfrak{A}_{2}$,

$$
=\sup \left\{\frac{\sum_{i, l=1}^{m} \sum_{j, k=1}^{n} \varphi_{1}\left[C_{i}^{*}\left(\alpha_{t}^{1} A_{j}^{1}\right)^{*}\left(\alpha_{t}^{1} A_{k}^{1}\right) C_{l}\right] \varphi_{2}\left[D_{i}^{*}\left(\alpha_{t}^{2} A_{j}^{2}\right)^{*}\left(\alpha_{t}^{2} A_{k}^{2}\right) D_{l}\right]}{\sum_{i, l} \varphi_{1}\left(C_{i}^{*} C_{l}\right) \varphi_{2}\left(D_{i}^{*} D_{l}\right)}\right\}^{1 / 2} .
$$

Let $E(\mathfrak{U})$ denote the set of states over $\mathfrak{A}$. We define automorphisms of $E(\mathfrak{A})$ by

$$
\tilde{\alpha} \varphi(A)=\varphi(\alpha A), \quad \alpha \in \text { Aut } \mathfrak{A} .
$$


Using this notation, we continue the above equation:

$=\sup \left\{\frac{\sum_{i, l} \sum_{j, k} \tilde{\alpha}_{t}^{1} \varphi_{1}\left[\left(\left(\alpha_{t}^{1}\right)^{-1} C_{i}\right)^{*} A_{j}^{1 *} A_{k}^{1}\left(\left(\alpha_{t}^{1}\right)^{-1} C_{l}\right)\right] \tilde{\alpha}_{t}^{2} \varphi_{2}\left[\left(\left(\alpha_{t}^{2}\right)^{-1} D_{i}\right)^{*} A_{j}^{2 *} A_{k}^{2}\left(\left(\alpha_{t}^{2}\right)^{-1} D_{l}\right)\right]}{\sum_{i, l} \tilde{\alpha}_{t}^{1} \varphi_{1}\left[\left(\left(\alpha_{t}^{1}\right)^{-1} C_{i}\right)^{*}\left(\left(\alpha_{t}^{1}\right)^{-1} C_{l}\right)\right] \tilde{\alpha}_{t}^{2} \varphi_{2}\left[\left(\left(\alpha_{t}^{2}\right)^{-1} D_{i}\right)^{*}\left(\left(\alpha_{t}^{2}\right)^{-1} D_{l}\right)\right]}\right\}^{1 / 2}$.

Instead of taking the supremum over all $\varphi_{i} \in E\left(\mathfrak{H}_{i}\right), i=1,2$, and all $C_{j} \in \mathfrak{A}_{1}$, $D_{j} \in \mathfrak{U}_{2}$, we may take it over all $\tilde{\alpha}_{t}^{i} \varphi_{i} \in E\left(\mathfrak{A}_{i}\right)$, all $\left(\alpha_{t}^{1}\right)^{-1} C_{j} \in \mathfrak{A}_{1}$, $\left(\alpha_{t}^{2}\right)^{-1} D_{j} \in \mathfrak{A}_{2}$, and we get

$$
\ldots=\left\|\Sigma A_{i}^{1} \otimes A_{i}^{2}\right\|_{\alpha} .
$$

Hence $\alpha_{t}^{1} \odot \alpha_{t}^{2}$ is norm-preserving and can be continued to an automorphism group $\alpha_{t}^{1} \otimes \alpha_{t}^{2}$ of $\mathfrak{A}_{1} \otimes_{\alpha} \mathfrak{A}_{2}$. Due to the continuity of $\Phi$, Eq. (1) can be extended to $\mathfrak{H}_{12}$ :

$$
\Phi \alpha_{t}=\alpha_{t}^{1} \otimes \alpha_{t}^{2} \Phi .
$$

Our main task will be to establish

Proposition 2.4. Let $\omega_{i}$ be $\beta$-K.M.S. states over $\mathfrak{A}_{i}$ with respect to $\alpha_{t}^{i}$, $i=1,2$. Then $\omega_{1} \otimes \omega_{2}$ is a $\beta$-K.M.S. state over $\mathfrak{I}_{1} \otimes_{\alpha} \mathfrak{A}_{2}$ with respect to $\alpha_{t}^{1} \otimes \alpha_{t}^{2}$.

With the help of this proposition, the proof of Theorem 2.3 is easily completed. Consider $\omega=\omega_{1} \otimes \omega_{2}{ }^{\circ} \Phi$. The continuity of $\omega\left(A \alpha_{t} B\right), A, B \in \mathfrak{A}_{12}$, is a trivial consequence of (2) and Proposition 2.4. Furthermore, for all $A, B \in \mathfrak{A}_{12}$ and all $f$ with $\mathscr{F} f \in \mathscr{D}$, we have

$$
\begin{aligned}
\int f(t-i \beta) \omega\left(A \alpha_{t} B\right) d t & =\int f(t-i \beta) \omega_{1} \otimes \omega_{2}\left[\Phi(A) \Phi\left(\alpha_{t} B\right)\right] d t \\
& =\int f(t-i \beta) \omega_{1} \otimes \omega_{2}\left[\Phi(A) \alpha_{t}^{1} \otimes \alpha_{t}^{2} \Phi(B)\right] d t \\
& =\int f(t) \omega_{1} \otimes \omega_{2}\left[\left(\alpha_{t}^{1} \otimes \alpha_{t}^{2} \Phi(B)\right) \Phi(A)\right] d t \\
& =\int f(t) \omega\left[\left(\alpha_{t} B\right) A\right] d t,
\end{aligned}
$$

where we again made use of Eq. (2) and Proposition 2.4. Therefore, $\omega$ is a $\beta$-K.M.S. state over $\mathfrak{A}_{12}$.

Remark 2.5. It would be desirable to define rather than assume the existence of an automorphism group $\alpha_{t}$ on $\mathfrak{A}_{12}$. It is easy to define $\alpha_{t}$ on $\mathfrak{A}_{1} \vee \mathfrak{A}_{2}$ by

$$
\alpha_{t} \Sigma A_{i}^{1} A_{i}^{2}=\Sigma\left(\alpha_{t}^{1} A_{i}^{1}\right)\left(\alpha_{t}^{2} A_{i}^{2}\right),
$$

or, equivalently, by

$$
\alpha_{t}=\Phi^{-1} \alpha_{t}^{1} \odot \alpha_{t}^{2} \Phi
$$

with the help of the isomorphism $\Phi: \mathfrak{A}_{1} \vee \mathfrak{H}_{2} \rightarrow \mathfrak{H}_{1} \odot \mathfrak{A}_{2}$. 
Unfortunately, it is not known whether $\Phi^{-1}$ is $\alpha$-norm continuous and can thus be extended to an isomorphism of $\mathfrak{H}_{1} \otimes_{\alpha} \mathfrak{I}_{2}$ onto $\mathfrak{A}_{12}$, thereby allowing to define $\alpha_{t}$ on $\mathfrak{A}_{12}$. Of course, if we assume that the composed system is described by $\mathfrak{A}_{1} \otimes_{\alpha} \mathfrak{I}_{2}$ then $(A)$ and $(E)$ are trivially fulfilled.

Now let us prove Proposition 2.4 .

In the following, $\mathfrak{I}, \mathfrak{I}_{1}, \mathfrak{I}_{2} \ldots$ etc. always denote $C^{*}$-algebras with time automorphisms $\alpha_{t}, \alpha_{t}^{1}, \alpha_{t}^{2} \ldots$ and states $\omega, \omega_{1}, \omega_{2} \ldots$

$\pi_{\omega}$ denotes the cyclic representation of $\mathfrak{A}$ given by $\omega, \alpha_{t}^{\omega}$ the corresponding representation of the time automorphisms $\alpha_{t}$, unitarily implemented by $U_{\omega}(t)$.

$\Phi_{A} \in \mathscr{H}_{\omega}$ is a vector of the representation space $\mathscr{H}_{\omega}$, defined by the equivalence class $[A]$ of $A \in \mathfrak{A}$. $\Omega_{\omega}$ is the cyclic vector such that $\omega(A)=\left(\Omega_{\omega}, \pi_{\omega}(A) \Omega_{\omega}\right)$.

Extensions of the state $\omega$ and the automorphisms $\alpha_{t}^{\omega}$ onto the von Neumann algebra $\mathfrak{B}_{\omega}$ generated by $\pi_{\omega}(\mathfrak{d})$ will be denoted by

$$
\tilde{\omega}(\cdot)=\left(\Omega_{\omega}, \Omega_{\omega}\right), \quad \tilde{\alpha}_{t}^{\omega} \cdot=U_{\omega}(t) \cdot U_{\omega}(t)^{-1} .
$$

To shorten the notation, we write $\tilde{\alpha}_{t}^{\omega_{t}}=\tilde{\alpha}_{t}^{i}, U_{\omega_{t}}(t)=U_{i}(t), \mathfrak{B}_{\omega_{t}}=\mathfrak{B}_{i}$.

In short, the proof of Proposition 2.4 is as follows: We consider the representations $\pi_{\omega_{i}}$ of $\mathfrak{A}_{i}$ given by the K.M.S. states $\omega_{i}$. The extensions $\tilde{\omega}_{i}$ are K.M.S. states over $\mathfrak{B}_{i}=\pi_{\omega_{i}}\left(\mathfrak{A}_{i}\right)^{\prime \prime}$. Let $\hat{\mathfrak{B}}_{i}$ denote the holomorphic elements of $\mathfrak{B}_{i}$. Due to the continuity in $t$ of $\omega_{i}\left(A \alpha_{t}^{i} B\right), \tilde{\alpha}_{t}^{i}$ are weakly continuous automorphisms of $\mathfrak{B}_{i}$ and therefore, (i) $\hat{\mathfrak{B}}_{i}$ is weakly dense in $\mathfrak{B}_{i}$, (ii) the direct algebraic product $\hat{\mathfrak{B}}_{1} \odot \hat{\mathfrak{B}}_{2}$ is weakly dense in $\left(\mathfrak{B}_{1} \otimes \mathfrak{B}_{2}\right)^{\prime \prime} . \tilde{\omega}_{1} \otimes \tilde{\omega}_{2}$ fulfills the condition (KMS) on $\hat{\mathfrak{B}}_{1} \odot \hat{\mathfrak{B}}_{2}$; due to the weak density of $\hat{\mathfrak{B}}_{1} \odot \hat{\mathfrak{B}}_{2}, \tilde{\omega}_{1} \otimes \tilde{\omega}_{2}$ is a K.M.S. state over $\left(\mathfrak{B}_{1} \otimes \mathfrak{B}_{2}\right)^{\prime \prime}$. However, $\left(\mathfrak{B}_{1} \otimes \mathfrak{B}_{2}\right)^{\prime \prime}$ $=\left(\pi_{\omega_{1}}\left(\mathfrak{A}_{1}\right) \otimes \pi_{\omega_{2}}\left(\mathfrak{A}_{2}\right)\right)^{\prime \prime}$ is isomorphic to $\pi_{\omega_{1} \otimes \omega_{2}}\left(\mathfrak{H}_{1} \otimes_{\alpha} \mathfrak{I}_{2}\right)^{\prime \prime}$, and hence $\omega_{1} \otimes \omega_{2}$ is a $\beta$-K.M.S. state over $\mathfrak{A}_{1} \otimes_{\alpha} \mathfrak{A}_{2}$.

Lemma 2.6. Let $\omega\left(A \alpha_{t} B\right)$ be a continuous function in $t$ for arbitrary $A, B \in \mathfrak{A}$. Then $\tilde{\alpha}_{t}$ is weakly continuous in $t:\left|\left(\Phi, \tilde{\alpha}_{t} C \Psi\right)\right| \underset{t \rightarrow 0}{\longrightarrow} 0$ for arbitrary $\Phi, \Psi \in \mathscr{H}_{\omega}, C \in \mathfrak{B}=\pi_{\omega}(\mathfrak{A})^{\prime \prime}$.

Proof. $U_{\omega}(t)$ is given by $U_{\omega}(t) \Phi_{A}=\Phi_{\alpha_{\star} A}, A \in \mathfrak{A}$. Any $\Phi, \Psi \in \mathscr{H}_{\omega}$ can be approximated by $\Phi_{A_{n}}$ and $\Psi_{B_{m}}$, respectively, with $\left\|\Phi_{A_{n}}\right\|=\|\Phi\|$, $\left\|\Psi_{B_{m}}\right\|=\|\Psi\|$. Hence

$$
\begin{aligned}
& \left|\left(\Phi,\left(U_{\omega}(t)-1\right) \Psi\right)\right| \\
& \leqq\left|\left(\Phi-\Phi_{A_{n}},\left(U_{\omega}(t)-1\right) \Psi\right)\right|+\mid\left(\Phi_{A_{n}},\left(U_{\omega}(t)-1\right)\left(\Psi-\Psi_{B_{m}}\right) \mid\right. \\
& +\left|\left(\Phi_{A_{n}},\left(U_{\omega}(t)-1\right) \Psi_{B_{m}}\right)\right| \\
& \leqq 2\left\|\Phi-\Phi_{A_{n}}\right\|\|\Psi\|+2\|\Phi\|\left\|\Psi-\Psi_{B_{m}}\right\|+\left|\omega\left(A_{n}^{*}\left(\alpha_{t}-1\right) B_{m}\right)\right|<\varepsilon
\end{aligned}
$$


for sufficiently large $n, m$ and sufficiently small $t$. Thus $U_{\omega}(t)$ is weakly continuous. It is also strongly continuous because it is unitary; and this trivially implies weak continuity of $\tilde{\alpha}_{t} C=U_{\omega}(t) C U_{\omega}(t)^{-1}, C \in \mathfrak{B}$.

Lemma 2.7. Let $\hat{\mathfrak{B}}$ denote the holomorphic elements of $\mathfrak{B}=\pi_{\omega}(\mathfrak{Q})^{\prime \prime}$. If $\alpha_{t}$ is weakly continuous, $\hat{\mathfrak{B}}$ is weakly dense in $\mathfrak{B}$.

Proof. For all $f(t)$ with Fourier transform $\mathscr{F} f \in \mathscr{D}$, we have

$$
B_{f}=\int f(t) \tilde{\alpha}_{t} B d t \in \hat{\mathfrak{B}}, \quad B \in \mathfrak{B},
$$

and there exists $t_{f}\left(\varepsilon_{1}\right)$ such that

$$
\left\|B_{f}-\int_{-t_{1}}^{t_{1}} f(t) \tilde{\alpha}_{t} B d t\right\|<\varepsilon_{1}, \quad \text { if } t_{1}>t_{f}\left(\varepsilon_{1}, B\right) .
$$

Let us choose a sequence $f_{r}(t), \mathscr{F} f_{r} \in \mathscr{D}$, such that $f_{r} \geqq 0, \int f_{r} d t=1, f_{r}$ converge to the $\delta$-function. Then $t_{f_{r}}\left(\varepsilon_{1}, B\right)$ can be made arbitrarily small ( $\varepsilon_{1}$ fixed). Due to the weak continuity of $\tilde{\alpha}_{t}$,

$$
\left|\left(\Phi,\left(\tilde{\alpha}_{t}-1\right) B \Psi\right)\right|<\varepsilon_{2}, \quad \text { if } \quad t<t_{0}\left(\varepsilon_{2} ; \Phi, \Psi, B\right) .
$$

Choose $r$ large enough such that $t_{f_{r}}\left(\varepsilon_{1}, B\right)<t_{0}\left(\varepsilon_{2} ; \Phi, \Psi, B\right)$, then

$$
\begin{aligned}
& \left|\left(\Phi,\left(B-B_{f_{r}}\right) \Psi\right)\right| \\
& \quad \leqq\left|\left(\Phi,\left(B-\int_{-t_{0}}^{t_{0}} f_{r}(t) \tilde{\alpha}_{t} B d t\right) \Psi\right)\right|+\left|\left(\Phi,\left(\int_{-t_{0}}^{t_{0}} f_{r}(t) \tilde{\alpha}_{t} B d t-B_{f_{r}}\right) \Psi\right)\right| \\
& \quad \leqq \varepsilon_{2}+\|\Phi\|\|\Psi\| \cdot \varepsilon_{1} .
\end{aligned}
$$

Lemma 2.8. If $\hat{\mathfrak{B}}_{i}, i=1,2$, are weakly dense in $\mathfrak{B}_{1}$ and $\mathfrak{B}_{2}$, respectively, then $\hat{\mathfrak{B}}_{1} \odot \hat{\mathfrak{B}}_{2}$ is weakly dense in $\left(\mathfrak{B}_{1} \otimes \mathfrak{B}_{2}\right)^{\prime \prime}$.

Proof. $\hat{\mathfrak{B}}_{1}$ and $\hat{\mathfrak{B}}_{2}$ are ${ }^{*}$-algebras, so we can use the density theorems of von Neumann and Kaplansky in order to conclude that any $B_{1} \in \mathfrak{B}$, and any $B_{2} \in \mathfrak{B}_{2}$ can be strongly approximated by nets $B_{1 \alpha} \in \hat{\mathfrak{B}}_{1}$ and $B_{2 \beta} \in \hat{\mathfrak{B}}_{2}$, respectively, with $\left\|B_{1 \alpha}\right\| \leqq\left\|B_{1}\right\|,\left\|B_{2 \beta}\right\| \leqq\left\|B_{2}\right\|$. Any $\Phi, \Psi \in \mathscr{H}$ can be approximated by vectors of the form

$$
\begin{gathered}
\Phi_{n}=\sum_{\text {finite }} \varphi_{1 i}^{n} \otimes \varphi_{2 i}^{n}, \quad \Psi_{n}=\sum_{\text {finite }} \psi_{1 j}^{n} \otimes \psi_{2 j}^{n} ; \\
\varphi_{1 i}^{n}, \psi_{1 j}^{n} \in \mathscr{H}_{1} ; \quad \varphi_{2 i}^{n}, \psi_{2 j}^{n} \in \mathscr{H}_{2} ; \quad\left\|\Phi_{n}\right\| \leqq\|\Phi\|,\left\|\Psi_{n}\right\| \leqq\|\Psi\| .
\end{gathered}
$$


Therefore,

$$
\begin{aligned}
\mid(\Phi,[ & {\left.\left[B_{1} \otimes B_{2}-B_{1 \alpha} \otimes B_{2 \beta}\right] \Psi\right) \mid } \\
\leqq & \left\|\Phi-\Phi_{n}\right\|\left\|B_{1} \otimes B_{2}-B_{1 \alpha} \otimes B_{2 \beta}\right\|\|\Psi\| \\
& +\left\|\Phi_{n}\right\|\left\|B_{1} \otimes B_{2}-B_{1 \alpha} \otimes B_{2 \beta}\right\|\left\|\Psi-\Psi_{n}\right\| \\
& +\left|\left(\Phi_{n},\left(B_{1}-B_{1 \alpha}\right) \otimes B_{2} \Psi_{n}\right)\right|+\left|\left(\Phi_{n}, B_{1 \alpha} \otimes\left(B_{2}-B_{2 \beta}\right) \Psi_{n}\right)\right| \\
\leqq & \left(\left\|\Phi-\Phi_{n}\right\| \cdot\|\Psi\|+\|\Phi\|\left\|\Psi-\Psi_{n}\right\|\right) \cdot 2\left\|B_{1}\right\|\left\|B_{2}\right\| \\
& +\sum_{i, j}\left(\varphi_{1 i}^{n},\left(B_{1}-B_{1 \alpha}\right) \psi_{1 j}^{n}\right)\left\|\varphi_{2 i}^{n}\right\|\left\|B_{2}\right\|\left\|\psi_{2 j}^{n}\right\| \\
& +\sum_{i, j}\left\|\varphi_{1 i}^{n}\right\|\left\|B_{1}\right\|\left\|\psi_{1 j}^{n}\right\| \|\left(\varphi_{2 i}^{n},\left(B_{2}-B_{2 \beta}\right) \psi_{2 j}^{n}\right) \mid,
\end{aligned}
$$

and this expression can be made arbitrarily small because the sums are finite. Hence any element $B_{1} \otimes B_{2}$ and thus any element $\sum_{\text {finite }} B_{1 i} \otimes B_{2 i}$ of $\mathfrak{B}_{1} \odot \mathfrak{B}_{2}$ can be weakly approximated by elements of $\mathfrak{B}_{1} \odot \hat{\mathfrak{B}}_{2}$; of course, $\mathfrak{B}_{1} \odot \mathfrak{B}_{2}$ is weakly dense in $\left(\mathfrak{B}_{1} \otimes \mathfrak{B}_{2}\right)^{\prime \prime}$. Q.E.D.

Lemma 2.9. Let $\tilde{\omega}_{1}$ and $\tilde{\omega}_{2}$ be $\beta$-K.M.S. states over $\mathfrak{B}_{1}$ and $\mathfrak{B}_{2}$ with respect to the automorphisms $\tilde{\alpha}_{t}^{1}, \tilde{\alpha}_{t}^{2}$, respectively. Then $\tilde{\omega}=\tilde{\omega}_{1} \otimes \tilde{\omega}_{2}$ fulfills the condition $\left(\mathrm{KMS}^{\prime}\right)$ on $\hat{\mathfrak{B}}_{1} \odot \mathfrak{\mathfrak { B }}_{2}$ with respect to the automorphisms $\tilde{\alpha}_{t}=\tilde{\alpha}_{t}^{1} \otimes \tilde{\alpha}_{t}^{2}$.

Proof. (KMS) implies (KMS'), thus for $B_{i}, C_{i} \in \hat{\mathfrak{B}}_{i}$ we have

$$
\tilde{\omega}_{1}\left(B_{1} \tilde{\alpha}_{i \beta} C_{1}\right)=\tilde{\omega}_{1}\left(C_{1} B_{1}\right) ; \quad \tilde{\omega}_{2}\left(B_{2} \tilde{\alpha}_{i \beta} C_{2}\right)=\tilde{\omega}_{2}\left(C_{2} B_{2}\right) .
$$

Take arbitrary elements $\sum_{\text {finite }} B_{1 k} \otimes B_{2 k}, \sum_{\text {finite }} C_{1 l} \otimes C_{2 l} \in \hat{\mathfrak{B}}_{1} \odot \hat{\mathfrak{B}}_{2}$, then

$$
\begin{aligned}
\tilde{\omega}_{1} \otimes \tilde{\omega}_{2}\left(\sum_{k} B_{1 k} \otimes B_{2 k}, \tilde{\alpha}_{i \beta}^{1} \otimes \tilde{\alpha}_{i \beta}^{2} \sum_{l} C_{1 l} \otimes C_{2 l}\right) \\
=\sum_{k, l} \tilde{\omega}_{1}\left(B_{1 k} \tilde{\alpha}_{i \beta}^{1} C_{1 l}\right) \tilde{\omega}_{2}\left(B_{2 k} \tilde{\alpha}_{i \beta}^{2} C_{2 l}\right) \\
=\sum_{k, l} \tilde{\omega}_{1}\left(C_{1 l} B_{1 k}\right) \tilde{\omega}_{2}\left(C_{2 l} B_{2 k}\right) \\
=\tilde{\omega}_{1} \otimes \tilde{\omega}_{2}\left(\sum_{l} C_{1 l} \otimes C_{2 l} \sum_{k} B_{1 k} \otimes B_{2 k}\right) .
\end{aligned}
$$

Proposition 2.10. Let $\omega_{1}, \omega_{2}$ be $\beta$-K.M.S. states over $\mathfrak{A}_{1}, \mathfrak{A}_{2}$ with respect to $\alpha_{t}^{1}$ and $\alpha_{t}^{2}$. The extensions of $\omega_{i}$ and $\alpha_{t}^{i}$ on $\mathfrak{B}_{i}=\pi_{\omega_{i}}\left(\mathfrak{H}_{i}\right)^{\prime \prime}$ will be denoted by $\tilde{\omega}_{i}$ and $\tilde{\alpha}_{t}^{i}$. Then $\tilde{\omega}=\tilde{\omega}_{1} \otimes \tilde{\omega}_{2}$ is a $\beta$-K.M.S. state over $\left(\mathfrak{B}_{1} \otimes \mathfrak{B}_{2}\right)^{\prime \prime}$ with respect to $\tilde{\alpha}_{t}=\tilde{\alpha}_{t}^{1} \otimes \tilde{\alpha}_{t}^{2}$. 
Proof. It is well known [1] that $\tilde{\omega}_{i}$ are $\beta$-K.M.S. states over $\mathfrak{B}_{i}$ with respect to $\tilde{\alpha}_{t}^{i}$. Therefore, we have, due to Lemma 2.9 and due to the equivalence of $\left(\mathrm{KMS}^{\prime}\right)$ and $(\mathrm{KMS})$ for holomorphic elements,

$$
\begin{gathered}
\tilde{\omega}\left(B \alpha_{t} C\right) \text { is continuous in } t, \quad B, C \in \hat{\mathfrak{B}}_{1} \odot \mathfrak{B}_{2}, \\
\int f(t-i \beta) \tilde{\omega}\left(B \tilde{\alpha}_{t} C\right) d t=\int f(t) \omega\left(\left(\tilde{\alpha}_{t} C\right) B\right) d t, \mathscr{F} f \in \mathscr{D}, B, C \in \hat{\mathfrak{B}}_{1} \odot \hat{\mathfrak{B}}_{2} .
\end{gathered}
$$

(i) The $\tilde{\omega}_{i}$ fulfill (KMS) (i), thus, according to Lemma 2.6, $\tilde{\alpha}_{t}^{i}$ are weakly continuous in $t$. The same is true for $\tilde{\alpha}_{t}: \tilde{\alpha}_{t} B=U(t) B U(-t)$, where $U(t)=U_{1}(t) \otimes U_{2}(t)$ is unitary and strongly continuous in $t$ since the $U_{i}(t)$ are strongly continuous. This implies that $\tilde{\alpha}_{t}$ is weakly continuous in $t$, and $\tilde{\omega}\left(B \tilde{\alpha}_{t} C\right)=\left(\Omega, B \tilde{\alpha}_{t} C \Omega\right)$ is continuous in $t$ for all $B, C \in\left(\mathfrak{B}_{1} \otimes \mathfrak{B}_{2}\right)^{\prime \prime}$, $\Omega=\Omega_{\omega_{1}} \otimes \Omega_{\omega_{2}}$; i.e. (3) is true for all $B, C \in\left(\mathfrak{B}_{1} \otimes \mathfrak{B}_{2}\right)^{\prime \prime}$.

(ii) Choose $C \in\left(\mathfrak{B}_{1} \otimes \mathfrak{B}_{2}\right)^{\prime \prime}, B \in \mathfrak{B}_{1} \odot \hat{\mathfrak{B}}_{2}$. According to Lemmas 2.6-2.8, $C$ is an element of the weak closure of $\hat{\mathfrak{B}}_{1} \odot \hat{\mathfrak{B}}_{2}$, and, since $\hat{\mathfrak{B}}_{1} \odot \hat{\mathfrak{B}}_{2}$ is an involutive algebra, also an element of the strong closure due to von Neumann's density theorem. Let $C_{\alpha} \in \hat{\mathfrak{B}}_{1} \odot \hat{\mathfrak{B}}_{2}$ converge strongly to $C$. Define

$$
\begin{array}{ll}
L_{\alpha}(t)=f(t-i \beta) \tilde{\omega}\left(B \tilde{\alpha}_{t} C_{\alpha}\right), & L(t)=f(t-i \beta) \tilde{\omega}\left(B \tilde{\alpha}_{t} C\right), \\
R_{\alpha}(t)=f(t) \omega\left(\left(\tilde{\alpha}_{t} C_{\alpha}\right) B\right), & R(t)=f(t) \omega\left(\left(\tilde{\alpha}_{t} C\right) B\right) .
\end{array}
$$

Then

$$
\begin{aligned}
\left|\int L(t) d t-\int L_{\alpha}(t) d t\right| & \leqq \int|f(t-i \beta)|\left|\tilde{\omega}\left(B \tilde{\alpha}_{t}\left(C-C_{\alpha}\right)\right)\right| d t \\
& \leqq \int|f(t-i \beta)|\left|\left(U(-t) B^{*} \Omega,\left(C-C_{\alpha}\right) \Omega\right)\right| d t \\
& \leqq \int|f(t-i \beta)| d t\left\|B^{*} \Omega\right\|\left\|\left(C-C_{\alpha}\right) \Omega\right\|<\varepsilon
\end{aligned}
$$

for sufficiently large $\alpha$.

A similar estimate holds for $\int\left(R(t)-R_{\alpha}(t)\right) d t$; because of $(4), \int L_{\alpha}(t) d t$ $=\int R_{\alpha}(t) d t$, and therefore, (4) holds for all $B \in \hat{\mathfrak{B}}_{1} \odot \hat{\mathfrak{B}}_{2}, C \in\left(\mathfrak{B}_{1} \otimes \mathfrak{B}_{2}\right)^{\prime \prime}$. Repeating the argument with respect to $B$, one realizes that (4) holds for arbitrary $B, C \in\left(\mathfrak{B}_{1} \otimes \mathfrak{B}_{2}\right)^{\prime \prime}$. Q.E.D.

Proposition 2.11. $\pi_{\omega_{1}}\left(\mathfrak{H}_{1}\right) \otimes \pi_{\omega_{2}}\left(\mathfrak{H}_{2}\right) \cong \pi_{\omega_{1} \otimes \omega_{2}}\left(\mathfrak{H}_{1} \otimes_{x} \mathfrak{A}_{2}\right)$.

(On the left hand side, " $\otimes$ " denotes the norm-closed tensor product, the norm is the operator norm in $\mathscr{H}_{\omega_{1}} \otimes \mathscr{H}_{\omega_{2}}$.)

Proof. (i) Define

$$
\begin{aligned}
& \mathscr{K}_{i}=\left\{A \in \mathfrak{H}_{i} ; \omega_{i}\left(A^{*} A\right)=0\right\}, \quad i=1,2 ; \\
& \mathscr{K}=\left\{A \in \mathfrak{H}_{1} \otimes_{\alpha} \mathfrak{H}_{2} ; \omega_{1} \otimes \omega_{2}\left(A^{*} A\right)=0\right\} ; \\
& \mathscr{D}_{i}=\mathfrak{V}_{i} / \mathscr{K}_{i}, i=1,2 ; \quad \mathscr{D}=\mathfrak{U}_{1} \odot \mathfrak{U}_{2} / \mathscr{K} .
\end{aligned}
$$


$\mathscr{D}_{1}, \mathscr{D}_{2}, \mathscr{D}$ are dense in $\mathscr{H}_{1}=\mathscr{H}_{\omega_{1}}, \mathscr{H}_{2}=\mathscr{H}_{\omega_{2}}, \mathscr{H}=\mathscr{H}_{\omega_{1} \otimes \omega_{2}}$, respectively. We introduce the mapping $f: \mathscr{D}_{1} \odot \mathscr{D}_{2} \rightarrow \mathscr{D}$ by $f\left(\sum_{k} \Phi_{A_{1 k}} \otimes \Phi_{A_{2 k}}\right)$ $=\Phi_{\Sigma A_{1 k} \otimes A_{2 k}}$, where $\Phi_{C}$ denotes the equivalence class of $C$. $f$ is well defined: take $B_{i k} \in \mathscr{K}_{i}, i=1,2$; then

with

$$
\Sigma\left(A_{1 k}+B_{1 k}\right) \otimes\left(A_{2 k}+B_{2 k}\right)=\Sigma A_{1 k} \otimes A_{2 k}+C
$$

$$
C=\Sigma A_{1 k} \otimes B_{2 k}+\Sigma B_{1 k} \otimes A_{2 k}+\sum B_{1 k} \otimes B_{2 k} \in \mathscr{K}
$$

and therefore,

$$
\begin{aligned}
\Phi_{A_{t k}} & =\Phi_{A_{t k}+B_{\imath k}}, \\
f\left(\sum \Phi_{A_{1 k}+B_{1 k}} \otimes \Phi_{A_{2 k}+B_{2 k}}\right) & =\Phi_{\Sigma\left(A_{1 k}+B_{1 k}\right) \otimes\left(A_{2 k}+B_{2 k}\right)} \\
& =\Phi_{\Sigma A_{1 k} \otimes A_{2 k}}=f\left(\Sigma \Phi_{A_{1 k}} \otimes \Phi_{A_{2 k}}\right) .
\end{aligned}
$$

Moreover, $f$ is isometric:

$$
\begin{aligned}
\left\|\Sigma \Phi_{A_{1 k}} \otimes \Phi_{A_{2 k}}\right\|^{2} & =\Sigma\left(\Phi_{A_{1 k}}, \Phi_{A_{1 l}}\right)_{1}\left(\Phi_{A_{2 k}}, \Phi_{A_{2 l}}\right)_{2}=\Sigma \omega_{1}\left(A_{1 k}^{*} A_{1 l}\right) \omega_{2}\left(A_{2 k}^{*} A_{2 l}\right) \\
& =\omega_{1} \otimes \omega_{2}\left(\left(\Sigma A_{1 k} \otimes A_{2 k}\right)^{*}\left(\Sigma A_{2 l} \otimes A_{2 l}\right)\right) \\
& =\left\|\Phi_{\Sigma A_{1 k} \otimes A_{2 k}}\right\|^{2} .
\end{aligned}
$$

Clearly, $f$ is a $1-1$-mapping of $\mathscr{D}_{1} \odot \mathscr{D}_{2}$ onto $\mathscr{D}$. Its domain and its range are dense in $\mathscr{H}_{1} \otimes \mathscr{H}_{2}$ and $\mathscr{H}$, respectively, hence it can be extended to an isometry $f: \mathscr{H}_{1} \otimes \mathscr{H}_{2} \rightarrow \mathscr{H}$. by

(ii) Now define the mapping $g: \pi_{\omega_{1}}\left(\mathfrak{A}_{1}\right) \odot \pi_{\omega_{2}}\left(\mathfrak{A}_{2}\right) \rightarrow \pi_{\omega_{1} \otimes \omega_{2}}\left(\mathfrak{A}_{1} \odot \mathfrak{A}_{2}\right)$

$$
g\left(\sum_{k} \pi_{\omega_{1}}\left(A_{1 k}\right) \otimes \pi_{\omega_{2}}\left(A_{2 k}\right)\right)=\pi_{\omega_{1} \otimes \omega_{2}}\left(\sum A_{1 k} \otimes A_{2 k}\right) .
$$

Clearly, $g$ is a $1-1$-mapping which respects the *-algebraic structure. $g$ is isometric: It is fairly easy to see that for $\Phi, \Psi \in \mathscr{D}_{1} \odot \mathscr{D}_{2}$ and $A \in \pi_{\omega_{1}}\left(\mathfrak{Q}_{1}\right) \odot \pi_{\omega_{2}}\left(\mathfrak{H}_{2}\right)$ we get

$$
(\Phi, A \Psi)=(f(\Phi), g(A) f(\Psi)),
$$

and therefore,

$$
\begin{aligned}
\|A\|^{2} & =\sup _{\Psi \in \mathscr{H}_{1} \otimes \mathscr{H}_{2}} \frac{\|A \Psi\|^{2}}{\|\Psi\|^{2}}=\sup _{\Psi \in \mathscr{D}_{1} \odot \mathscr{D}_{2}} \frac{\left(\Psi, A^{*} A \Psi\right)}{(\Psi, \Psi)} \\
& =\sup _{\Psi \in \mathscr{D}_{1} \odot \mathscr{D}_{2}} \frac{\left(f(\Psi), g\left(A^{*} A\right) f(\Psi)\right)}{(f(\Psi), f(\Psi))} \\
& =\sup _{f(\Psi) \in \mathscr{D}} \frac{\left(f(\Psi), g(A)^{*} g(A) f(\Psi)\right)}{(f(\Psi), f(\Psi))}=\|g(A)\|^{2} .
\end{aligned}
$$


Thus $g$ can be extended to the norm closure of $\pi_{\omega_{1}}\left(\mathfrak{H}_{1}\right) \odot \pi_{\omega_{2}}\left(\mathfrak{A}_{2}\right)$, and we get an isomorphism

$$
g: \pi_{\omega_{1}}\left(\mathfrak{A}_{1}\right) \otimes \pi_{\omega_{2}}\left(\mathfrak{A}_{2}\right) \rightarrow \pi_{\omega_{1} \otimes \omega_{2}}\left(\mathfrak{H}_{1} \otimes_{\alpha} \mathfrak{H}_{2}\right) . \quad \text { Q.E.D. }
$$

Remark 2.12. (i) Eq. (5) holds for arbitrary $\Phi, \Psi \in \mathscr{H}_{1} \otimes \mathscr{H}_{2}$, and arbitrary $A \in \pi_{\omega_{1}}\left(\mathfrak{A}_{1}\right) \otimes \pi_{\omega_{2}}\left(\mathfrak{A}_{2}\right)$ because both $f$ and $g$ are isometric.

(ii) By construction of $f$, we have $f\left(\Omega_{\omega_{1}} \otimes \Omega_{\omega_{2}}\right)=\Omega_{\omega_{1} \otimes \omega_{2}}$.

(iii) By construction of $g$ and due to its continuity, we have

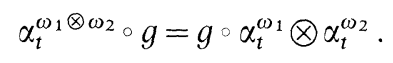

Combining the results of the preceeding propositions we can easily achieve the

Proof of Proposition 2.4. For any $A, B \in \mathfrak{A}_{1} \otimes_{\alpha} \mathfrak{I}_{2}$ :

$$
\omega_{1} \otimes \omega_{2}\left(A \alpha_{t}^{1} \otimes \alpha_{t}^{2} B\right)=\left(\Omega_{\omega_{1} \otimes \omega_{2}}, \pi_{\omega_{1} \otimes \omega_{2}}(A) \alpha_{t}^{\omega_{1} \otimes \omega_{2}} \pi_{\omega_{1} \otimes \omega_{2}}(B) \Omega_{\omega_{1} \otimes \omega_{2}}\right),
$$

and, according to (5) and Remark 2.12:

$$
\begin{aligned}
& =\left(\Omega_{\omega_{1}} \otimes \Omega_{\omega_{2}}, g^{-1}\left(\pi_{\omega_{1} \otimes \omega_{2}}(A)\right) \alpha_{t}^{\omega_{1}} \otimes \alpha_{t}^{\omega_{2}} g^{-1}\left(\pi_{\omega_{1} \otimes \omega_{2}}(B)\right) \Omega_{\omega_{1}} \otimes \Omega_{\omega_{2}}\right) \\
& =\tilde{\omega}_{1} \otimes \tilde{\omega}_{2}\left(A^{\prime} \tilde{\alpha}_{t}^{1} \otimes \tilde{\alpha}_{t}^{2} B^{\prime}\right)
\end{aligned}
$$

with $A^{\prime}=g^{-1}\left(\pi_{\omega_{1} \otimes \omega_{2}}(A)\right), B^{\prime}=g^{-1}\left(\pi_{\omega_{1} \otimes \omega_{2}}(B)\right) \in\left(\mathfrak{B}_{1} \otimes \mathfrak{B}_{2}\right)^{\prime \prime}$. Proposition 2.10 tells us that $\tilde{\omega}_{1} \otimes \tilde{\omega}_{2}$ fulfills the condition (KMS), therefore, $\omega_{1} \otimes \omega_{2}$ is a K.M.S. state over $\mathfrak{A}_{1} \otimes_{\alpha} \mathfrak{H}_{2}$. Q.E.D.

\section{Thermal Coupling of Finite Quantum Systems}

It cannot be seen from the formalism of the last section, whether or not the systems under consideration are coupled thermally. In classical thermodynamics, thermal contact of two systems is an interaction of the systems, which allows energy transfer without particle exchange, without momentum transfer and without a change of the volumes of the systems. We have to show how in the case of quantum systems thermal coupling can be introduced in such a way that the assumptions of Section II remain unchanged if the thermal interaction is switched on, provided the temperatures of the systems are the same. However, to achieve this goal it is necessary to deal with a less abstract situation. We shall regard our systems as limits of finite systems in finite boxes and try to construct an interaction satisfying the above requirements. We shall disregard the momentum transfer which, in our model, will be zero at least at equal temperatures. 
Especially, we want to preserve the property $\alpha_{t}^{i} \mathfrak{A}_{i} \subset \mathfrak{A}_{i}, i=1,2$. The easiest way to achieve this is the use of a classical system $S_{c}$ interacting with both quantum systems $S_{1}$ and $S_{2}$, representing a heat transferring part of the walls of the boxes. This may be justified by remarking that heat exchange is normally a very slow process compared to processes on an atomic level.

We start with finite quantum systems $S_{1}^{\Lambda_{1}}$ and $S_{2}^{\Lambda_{2}}$ in finite regions $\Lambda_{1}$ and $\Lambda_{2}$ respectively, $\Lambda_{1} \cap \Lambda_{2}=\emptyset$. The $S_{i}^{\Lambda_{i}}$ shall be described by Hamiltonians $H_{i}$ acting in Hilbert spaces $\mathscr{H}_{i}, i=1,2$. The Hilbert space of the composite system is $\mathscr{H}=\mathscr{H}_{1} \otimes \mathscr{H}_{2}$. (We identify $H_{1}$ with $H_{1} \otimes \mathbf{1}_{2}$ and $H_{2}$ with $\mathbf{1}_{1} \otimes H_{2}$.) $S_{c}$ is given by a classical Hamiltonian $H_{c}\left(\underline{p}_{c}, q_{c}\right)$, where $\underline{p}_{c}$ and $q_{c}$ denote finite sets of canonical variables. To formulate the interaction we use the Schrödinger picture. The total Hamiltonian is

$$
\begin{aligned}
H=H_{1}\left(\underline{p}_{1}, \underline{q}_{1}\right)+W_{1}\left(\underline{p}_{1}, q_{1} ; \underline{p}_{c}, \underline{q}_{c}\right)+H_{2}\left(\underline{p}_{2}, \underline{q}_{2}\right) & +W_{2}\left(\underline{p}_{2}, \underline{q}_{2} ; \underline{p}_{c}, \underline{q}_{c}\right) \\
& +H_{c}\left(\underline{p}_{c}, q_{c}\right) \cdot \mathbf{1},
\end{aligned}
$$

where $q_{i}, i=1,2$, are finite sets of variables, $\underline{p}_{i}=\frac{\partial}{\partial q_{i}} ; W_{i}$ acts in $\mathscr{H}_{i}$, and $\mathbf{1}$ is the identity in $\mathscr{H}$. To prevent unnecessary complications, we assume $W_{i}$ to be self-adjoint bounded operators, $\left\|W_{i}\right\| \leqq c$. This ensures, with the aid of Kato's Theorem [14], that $H$ is self-adjoint.

The equations of motion are

$$
\begin{aligned}
& i \frac{\partial}{\partial t} \psi\left(q_{1}, q_{2}, t\right)=H \psi\left(q_{1}, q_{2}, t\right), \\
& \frac{d}{d t} \underline{p}_{c}=-\frac{\partial\langle H\rangle}{\partial \underline{q}_{c}}=-\frac{\partial H_{c}}{\partial \underline{q}_{c}}-\frac{\partial\langle W\rangle}{\partial \underline{q}_{c}}, \\
& \frac{d}{d t} q_{c}=\frac{\partial\langle H\rangle}{\partial \underline{p}_{c}}=\frac{\partial H_{c}}{\partial \underline{p}_{c}}+\frac{\partial\langle W\rangle}{\partial \underline{p_{c}}},
\end{aligned}
$$

with $\langle A\rangle=(\psi, A \psi)$ denoting the expectation value of an operator $A$ in the state determined by Eq. (7), $W=W_{1}+W_{2}$.

Eq. (7) admits a product solution

$$
\psi\left(q_{1}, \underline{q}_{2}, t\right)=e^{-i \int_{t_{0}}^{t} H_{c}\left(\underline{p}_{c}\left(t^{\prime}\right), \underline{q}_{c}\left(t^{\prime}\right)\right) d t^{\prime}} \psi_{1}\left(\underline{q}_{1}, t\right) \psi_{2}\left(\underline{q}_{2}, t\right),
$$

where the $\psi_{i}$ obey Schrödinger equations with Hamiltonians $H_{i}+W_{i}$.

This separation of variables corresponds to our requirement that $\alpha_{t}^{i} \mathfrak{A}_{i} \subset \mathfrak{A}_{i}$. Eqs. (7) and (8) are time translation invariant, hence $\langle H\rangle$ is a constant of motion (but this is no longer true for the operator $H$ !). This can be checked by direct computation. 
From (7) and (8) we calculate

$$
\begin{aligned}
\frac{d}{d t}\left\langle H_{k}\right\rangle & =i\left\langle\left[H, H_{k}\right]\right\rangle=i\left\langle\left[W_{k}, H_{k}\right]\right\rangle, \quad k=1,2 ; \\
\frac{d}{d t}\left\langle H_{k}+W_{k}\right\rangle & =i\left\langle\left[H, H_{k}+W_{k}\right]\right\rangle+\frac{\partial\left\langle W_{k}\right\rangle}{\partial \underline{q}_{c}} \frac{d \underline{q}_{c}}{d t}+\frac{\partial\left\langle W_{k}\right\rangle}{\partial \underline{p}_{c}} \frac{d \underline{p}_{c}}{d t} \\
& =\frac{\partial\left\langle W_{k}\right\rangle}{\partial \underline{q}_{c}} \frac{\partial\langle H\rangle}{\partial \underline{p}_{c}}-\frac{\partial\left\langle W_{k}\right\rangle}{\partial \underline{p}_{c}} \frac{\partial\langle H\rangle}{\partial \underline{q}_{c}} \\
\frac{d}{d t} H_{c} & =\frac{\partial H_{c}}{\partial \underline{q}_{c}} \frac{\partial\langle W\rangle}{\partial \underline{p} \underline{p}_{c}}-\frac{\partial H_{c}}{\partial \underline{p}_{c}} \frac{\partial\langle W\rangle}{\partial \underline{q}_{c}} .
\end{aligned}
$$

By these equations it is shown that, in general, neither $\left\langle H_{k}\right\rangle$ nor $\left\langle H_{k}+W_{k}\right\rangle$ are constant, therefore, the interaction admits energy transfer from one quantum system to the other, provided $W_{k}$ and $H_{k}$ do not commute.

The classical system $S_{c}$ represents the "wall" between the quantum systems through which the heat exchange can take place. In classical thermodynamics, it is understood that the wall itself does not contain, or store, energy. In order to approximate this situation, we have to consider a special case: we sandwich $S_{c}$ between like quantum systems with opposite signs of the interactions:

with

$$
\begin{gathered}
\Lambda_{1}=\Lambda, \Lambda_{2}=\Lambda+a \quad \text { such that } \quad \Lambda \cap(\Lambda+a)=\emptyset ; \\
H_{1}=\tilde{H} \otimes \mathbf{1}, \quad W_{1}=V\left(\underline{p}_{c}, \underline{q}_{c}\right) \otimes \mathbf{1} ; \\
H_{2}=\mathbf{1} \otimes \tilde{H}, \quad W_{2}=-\mathbf{1} \otimes V\left(\underline{p}_{c}, \underline{q}_{c}\right) ;
\end{gathered}
$$

Here $p_{0}, q_{0}$ are solutions of the unperturbed classical system given by $H_{c}$; in $W_{1}$ and $W_{2}$ we have suppressed the respective variables $\underline{p}_{1}, q_{1}$ and $\underline{p}_{2}, q_{2}$.

Preferring to describe $S_{1}$ and $S_{2}$ in the Heisenberg picture, we have to look for the automorphisms $\alpha_{t}$ of time translations and put

$$
\langle\cdot\rangle=\omega\left(\alpha_{t} \cdot\right) .
$$

Since $H$ generally depends on $t$ through the time dependence of $\underline{p}_{c}$ and $q_{c}, \alpha_{t}$ is not a group of automorphisms. Formally, we can write

$$
\alpha_{t} A=U(t) A U(t)^{-1}, \quad U(t)=T e^{i \int_{0}^{t} H\left(t^{\prime}\right) d t^{\prime}},
$$

the symbol $T$ denoting time ordering.

In order to introduce the temperature, we consider $S_{1}$ and $S_{2}$ as canonical ensembles. In the unperturbed case, their equilibrium states 
$\omega_{1}$ and $\omega_{2}$ are given by density matrices

$$
\varrho_{i}=\frac{e^{-\beta_{\imath} H_{i}}}{\operatorname{Tr} e^{-\beta_{t} H_{l}}}, \quad \omega_{i}(\cdot)=\operatorname{Tr}\left(\varrho_{i} \cdot\right) ;
$$

the unperturbed time automorphisms are

$$
\alpha_{t}^{i} A=e^{i H_{2} t} A e^{-i H_{2} t}, \quad i=1,2 .
$$

Now let us switch on the interactions $W_{1}$ and $W_{2}$. A general solution cannot be given here. We are interested in a special one which leaves the systems essentially unchanged. As long as $\beta_{1}=\beta_{2}$, such a solution exists:

$\left.\begin{array}{l}\alpha_{t} A=U(t) A U(t)^{-1}, U(t)=\exp i\left(H_{1}+W_{1}\left(\underline{p}_{0}, \underline{q}_{0}\right)+H_{2}+W_{2}\left(\underline{p}_{0}, \underline{q}_{0}\right)\right) \cdot t ; \\ \underline{p}_{c}=\underline{p}_{0}, \underline{q}_{c}=q_{0} ; \\ \omega=\omega_{1} \otimes \omega_{2}, \omega_{i}=\operatorname{Tr}\left(\varrho_{i}, \cdot\right), \varrho_{i}=\frac{\exp \left[-\beta\left(H_{i}+W_{i}\left(\underline{p}_{0}, \underline{q}_{0}\right)\right)\right]}{\operatorname{Tr} \exp \left[-\beta\left(H_{i}+W_{i}\left(\underline{p}_{0}, \underline{q}_{0}\right)\right)\right]} .\end{array}\right\}$

$\left(e^{-\beta H_{l}}\right.$ are trace class operators, and so are $e^{-\beta\left(H_{l}+W_{\imath}\right)}$ due to the boundedness of $W_{i}$.)

This is a solution since

$$
\omega\left(\alpha_{t}\left(W_{1}+W_{2}\right)\right)=\omega\left(W_{1}+W_{2}\right)=0
$$

due to (9) and (10), and hence $\underline{p}_{c}$ and $\underline{q}_{c}$ obey the unperturbed classical equations given by $H_{c}$. This in turn leaves $W_{i}\left(\underline{p}_{c}, \underline{q}_{c}\right)=W_{i}\left(\underline{p}_{0}, q_{0}\right)$ time invariant according to (11), so that $\alpha_{t}$ is indeed given by the above expression. As a simple consequence we have $\frac{d}{d t}\left\langle H_{k}+W_{k}\right\rangle=0$.

Of course, what is done here, is neither a proof that systems in thermal equilibrium must have equal temperatures, nor a complete model of heat exchange. This would require a theory describing how a system approaches its equilibrium state. Our point is to give an argument that the assumptions of Section II can be maintained in the presence of thermal contact in the case of equal temperatures: we have put forward a simple model of thermal interaction and given a solution of the desired type such that the assumptions $(A)$ to $(E)$ can be satisfied.

\section{Coupling of Infinite Systems}

We are interested in thermally coupling infinite systems, so let us consider the infinite volume limit

$$
\Lambda_{i} \rightarrow \infty, \quad \frac{n_{i}}{V_{i}} \rightarrow \varrho_{i}, \quad i=1,2,
$$


of our model systems, where $V_{i}=$ volume of the region $\Lambda_{i}, n_{i}=$ number of particles. The properties which are important for applying the theorem of Section II, namely the existence of uncoupled solutions

$$
\omega=\omega_{1} \otimes \omega_{2}, \quad \alpha_{t}=\alpha_{t}^{1} \otimes \alpha_{t}^{2}
$$

modified only by replacement of $H_{i}$ by $H_{i}+W_{i}$ with constant $W_{i}$ if $\beta_{1}=\beta_{2}$, are evidently not affected by taking the limit. But we have to assure ourselves that switching on and off the thermal coupling acts continuously on the systems and can be interchanged with the infinite volume limit. Therefore, let us consider a set of systems $\left(\mathfrak{H}_{A}, \omega_{\lambda}^{\Lambda}, \alpha_{t}^{\Lambda}(\lambda)\right)$ with

$$
\begin{gathered}
\omega_{\lambda}^{\Lambda}(A)=\frac{\operatorname{Tr} e^{-\beta(H(A)+\lambda W)} A}{\operatorname{Tr} e^{-\beta(H(\Lambda)+\lambda W)}}, \quad A \in \mathfrak{A}_{\Lambda} \\
\alpha_{t}^{\Lambda}(\lambda) A=U_{\lambda}^{\Lambda}(t) A U_{\lambda}^{\Lambda}(t)^{-1}, \quad U_{\lambda}^{\Lambda}(t)=e^{i(H(A)+\lambda W) t}, \quad A \in \mathfrak{H}_{\Lambda} .
\end{gathered}
$$

The following questions have to be answered:

1) Is $\alpha_{t}^{\Lambda}(\lambda)$ continuous in $\lambda$ at $\lambda=0$ ?

2) Does continuity hold in the limit $\Lambda \rightarrow \infty$ ?

3) Are the states $\omega_{\lambda}^{\Lambda}$ continuous in $\lambda$ and what about the infinite volume limit?

4) How is the limit state $\omega$ affected by the introduction of the interaction $W$ ?

Since $W$ is intended to represent the interaction of the system with parts of its boundary, we assume

(F) $W$ is given by $m$-particle potentials, $m=1,2, \ldots m_{0}$, with support in the region $\Lambda_{0},\|W\| \leqq c$.

$\Lambda_{0}$ will be held fixed in the infinite volume limit. Therefore, neither the temperedness nor, for sufficiently small $c$, the stability of the total potential will be disturbed by switching on the interaction, if the particleparticle-interactions in $H(\Lambda)$ are given by tempered and stable potentials.

1. The first question is answered by

Proposition 4.1. Let the automorphism group $\alpha_{t}(\lambda)$ of the operator- $C^{*}-$ algebra $\mathfrak{A}$ be implemented by $e^{i(H+\lambda W) t}$, where $H$ and $W$ are self-adjoint operators, $W$ bounded. Then $\alpha_{t}(\lambda)$ is strongly continuous in $\lambda$ for fixed $t$.

Proof. Theorem IX.2.1 of [14] tells us that $e^{i(H+\lambda W) t}$ is, for fixed $t$, an entire function of $\lambda$ and therefore norm-continuous in $\lambda$. Hence

$$
\begin{aligned}
\| \alpha_{t}(\lambda) A & -\alpha_{t}\left(\lambda^{\prime}\right) A\|\leqq\|\left(e^{i(H+\lambda W) t}-e^{i\left(H+\lambda^{\prime} W\right) t}\right) A e^{-i(H+\lambda W) t} \| \\
& +\left\|e^{i\left(H+\lambda^{\prime} W\right) t} A\left(e^{-i(H+\lambda W) t}-e^{-i\left(H+\lambda^{\prime} W\right) t}\right)\right\| \\
& \leqq 2 \varepsilon\|A\|
\end{aligned}
$$

if $\left|\lambda-\lambda^{\prime}\right|$ is sufficiently small. Q.E.D. 
Especially, we have continuity at $\lambda=0$.

2. To answer the second question, we have to replace $H$ by $H(\Lambda)$. We write $\alpha_{t}^{\Lambda}(\lambda)$ to indicate the $\Lambda$-dependence of the automorphisms, defined as above. Now let us suppose that, for fixed $\lambda$, the limit $\lim _{\lambda \rightarrow \infty} \alpha_{t}^{A}(\lambda)=\alpha_{t}(\lambda)$ exists and defines a group of automorphisms of the global algebra $\mathfrak{A}$ of observables. There is no general proof for the existence of this limit. Robinson [15] has given a proof for the case of quantum lattice systems with sufficiently nice potentials. The class of those potentials is large enough to be invariant under the addition of a "thermal" interaction potential as specified by (F). We can expect that to be true whenever one can exhibit a class of nice potentials and show the existence of the limit $\lim _{\Lambda \rightarrow \infty} \alpha_{t}^{\Lambda}$ for $\lambda=0$.

From the next proposition, we see that $\alpha_{t}(\lambda)$ too is continuous in $\lambda$.

Proposition 4.2. Let $\alpha_{t}\left(H_{\imath}, \lambda\right), \imath \in I$, be a set of automorphism groups of $\mathfrak{A}$ implemented by $e^{i\left(H_{t}+\lambda W\right) t} ; H_{\imath}$ self-adjoint, $W$ self-adjoint and bounded. Then $\alpha_{t}\left(H_{l}, \lambda\right)$ is strongly continuous in $\lambda$ for fixed $t$ uniformly in $l \in I$.

Proof. It suffices to show that the norm-continuity of $e^{i\left(H_{2}+\lambda W\right) t}$, established in Theorem IX.2.1 of [14], is uniform in $l$. Looking at the proof of this theorem, we see that $e^{i\left(\boldsymbol{H}_{2}+\lambda W\right) t}$ can be written as

$$
e^{i\left(H_{l}+\lambda W\right) t}=\sum_{n=0}^{\infty} U_{\imath n}^{\lambda}(t),
$$

where the $U$ 's are $n$-linear in $\lambda W$ and bounded by

$$
\left\|U_{i n}^{\lambda}(t)\right\| \leqq\|\lambda W\|^{n} \frac{t^{n}}{n !} .
$$

In general, there are two additional factors $M_{l}^{n+1} e^{\gamma_{2} t}$ on the right hand side of (14), $M_{\imath}$ and $\gamma_{\imath}$ depending on the class $\mathscr{G}\left(M_{\imath}, \gamma_{\imath}\right)$ to which $i H_{\imath}$ belongs (for the notation compare [14]). But for all self-adjoint operators and hence for all $H_{\imath}, \imath \in \mathrm{I}$, we have

$$
\pm i H_{\imath} \in \mathscr{G}(1,0) \text {. }
$$

Due to (14), the series (13) converges uniformly in $l$ for fixed $t$ and so we get uniform continuity of $e^{i\left(H_{l}+\lambda W\right) t}$. Q.E.D.

3. Let us consider states $\omega_{\lambda}^{A}$ as defined in the third question. It is tacitly assumed that $e^{-\beta H(\Lambda)}$ is a trace class operator. Because of the boundedness of $W$, the same is true of $e^{-\beta(H(\Lambda)+\lambda W)}$. Let us introduce the abbreviation $E_{\lambda}^{\Lambda}(\beta)=e^{-\beta(H(A)+\lambda W)}$; and let $\|\ldots\|_{1}$ denote the trace norm defined by $\|A\|_{1}=\operatorname{Tr}\left(\left(A^{*} A\right)^{1 / 2}\right)$. Then we get

Lemma 4.3. $\left\|E_{\lambda}^{\Lambda}(\beta)\right\|_{1} \leqq e^{\beta|\lambda| c}\left\|E_{0}^{\Lambda}(\beta)\right\|_{1}$, if $\lambda$ is real. 
Proof. Recall that $W$ is supposed to be self-adjoint. Let $\varepsilon_{m}^{A}(\lambda)$ be the $m$-th eigenvalue of $H(\Lambda)+\lambda W$ (in increasing order and repeated according to multiplicity). $\|W\| \leqq c$ implies

$$
\begin{aligned}
\varepsilon_{m}^{A}(\lambda) & \geqq \varepsilon_{m}^{\Lambda}(0)-|\lambda| c \\
\left\|E_{\lambda}^{A}(\beta)\right\|_{1} & =\operatorname{Tr} e^{-\beta(H(A)+\lambda W)}=\sum_{m} e^{-\beta \varepsilon_{m}^{A}(\lambda)} \\
& \leqq \sum e^{-\beta \varepsilon_{m}^{A}(0)+\beta|\lambda| c} \leqq e^{\beta|\lambda| c}\left\|E_{0}^{\Lambda}(\beta)\right\|_{1} .
\end{aligned}
$$

Now we want to establish

Proposition 4.4. The state $\omega_{\lambda}^{\Lambda}$ defined by $\omega_{\lambda}^{\Lambda}(A)=\frac{\operatorname{Tr}\left(E_{\lambda}^{\Lambda}(\beta) A\right)}{\operatorname{Tr} E_{\lambda}^{\Lambda}(\beta)}$ with $E_{\lambda}^{\Lambda}(\beta)$ as above, $\|W\| \leqq c$, depends norm-continuously on $\lambda$.

Proof. The essential point is to show the trace-norm continuity in $\lambda$ of $E_{\lambda}^{\Lambda}(\beta)$. For fixed $\Lambda, E_{0}^{\Lambda}(\beta)$ can be considered as an element of the semigroup $\left\{E_{0}^{\Lambda}(\xi), \xi>0\right\}$, generated by $H(\Lambda)$. Since $H(\Lambda)$ is self-adjoint and bounded below, $H(\Lambda) \geqq-\gamma_{\Lambda}$, we have

$$
\left\|E_{0}^{A}(\xi)\right\| \leqq e^{\gamma_{A} \xi} \text {. }
$$

Introducing the bounded perturbation $\lambda W$, we get another semigroup $\left\{E_{\lambda}^{\Lambda}(\xi), \xi>0\right\}$ with

$$
\left\|E_{\lambda}^{\Lambda}(\xi)\right\| \leqq e^{\left(\gamma_{\Lambda}+|\lambda| c\right) \xi},
$$

compare Theorem IX.2.1 of [14]. According to the proof of the cited theorem, $E_{\lambda}^{\Lambda}(\xi)$ satisfies the following integral equation:

Therefore,

$$
E_{\lambda}^{\Lambda}(\xi)=E_{0}^{\Lambda}(\xi)-\int_{0}^{\xi} E_{0}^{\Lambda}\left(\xi-\xi^{\prime}\right) \lambda W E_{\lambda}^{\Lambda}\left(\xi^{\prime}\right) d \xi^{\prime} .
$$

$$
\begin{aligned}
\left\|E_{\lambda}^{\Lambda}(\xi)-E_{0}^{\Lambda}(\xi)\right\|_{1} & \leqq \int_{0}^{\xi}\left\|E_{0}^{\Lambda}\left(\xi-\xi^{\prime}\right) \lambda W E_{\lambda}^{\Lambda}(\xi)\right\|_{1} d \xi^{\prime} \\
& \leqq \int_{0}^{\xi / 2}\left\|E_{0}^{\Lambda}\left(\xi-\xi^{\prime}\right)\right\|_{1}\|\lambda W\|\left\|E_{\lambda}^{\Lambda}\left(\xi^{\prime}\right)\right\| d \xi^{\prime} \\
& +\int_{\xi / 2}^{\xi}\left\|E_{0}^{\Lambda}\left(\xi-\xi^{\prime}\right)\right\|\|\lambda W\|\left\|E_{\lambda}^{\Lambda}\left(\xi^{\prime}\right)\right\|_{1} d \xi^{\prime} .
\end{aligned}
$$

Using Lemma 4.3 and inserting (16) and $\left(16^{\prime}\right)$ we get

$$
\begin{aligned}
& \left\|E_{\lambda}^{\Lambda}(\xi)-E_{0}^{\Lambda}(\xi)\right\|_{1} \\
& \quad \leqq|\lambda| c\left(\int_{0}^{\xi / 2}\left\|E_{0}^{\Lambda}\left(\xi-\xi^{\prime}\right)\right\|_{1} e^{\left(\gamma_{1}+|\lambda| c\right) \xi^{\prime}} d \xi^{\prime}+\int_{\xi / 2}^{\xi} e^{\gamma_{\Lambda}\left(\xi^{\xi}-\xi^{\prime}\right)} e^{|\lambda| c \xi^{\prime}}\left\|E_{0}^{\Lambda}(\xi)\right\|_{1} d \xi^{\prime}\right) \\
& \quad \leqq|\lambda| c\left(e^{|\lambda| c \xi^{\prime} / 2}+e^{|\lambda| c \xi^{\zeta}}\right) \cdot J_{0}^{\Lambda}
\end{aligned}
$$


with

$$
J_{0}^{\Lambda}=\int_{0}^{\xi / 2}\left\|E_{0}^{\Lambda}\left(\xi-\xi^{\prime}\right)\right\|_{1} e^{\gamma_{\Lambda} \xi^{\prime}} d \xi^{\prime}=\int_{0}^{\xi / 2} \sum_{m} e^{-\left(\varepsilon_{m}^{A}(0)+\gamma_{\Lambda}\right)\left(\xi^{\prime}-\xi^{\prime}\right)} e^{\gamma_{\Lambda} \xi} d \xi^{\prime} .
$$

Since $\varepsilon_{m}^{\Lambda}+\gamma_{\Lambda} \geqq 0$ because of $H(\Lambda) \geqq-\gamma_{\Lambda}$, the integrand reaches its maximum for $\xi^{\prime}=\xi / 2$; hence

$$
\begin{gathered}
J_{0}^{\Lambda} \leqq e^{\gamma_{\Lambda} \xi} \sum_{m} e^{-\left(\varepsilon_{m}^{A}(0)+\gamma_{\Lambda}\right) \xi / 2} \cdot \xi / 2=e^{\gamma_{\Lambda} \xi / 2}\left\|E_{0}^{\Lambda}(\xi / 2)\right\|_{1} \cdot \xi / 2, \\
\left\|E_{\lambda}^{\Lambda}(\xi)-E_{0}^{\Lambda}(\xi)\right\|_{1} \leqq|\lambda| c \cdot 2 \xi e^{\gamma_{\Lambda} \xi / 2}\left\|E_{0}^{\Lambda}(\xi / 2)\right\|_{1}
\end{gathered}
$$

for sufficiently small $\lambda$.

Now we can compute

$$
\begin{gathered}
\left|\omega_{\lambda}^{\Lambda}(A)-\omega_{0}^{\Lambda}(A)\right|=\frac{\operatorname{Tr} E_{\lambda}^{\Lambda}(\beta) A \cdot \operatorname{Tr} E_{0}^{\Lambda}(\beta)-\operatorname{Tr} E_{0}^{\Lambda}(\beta) A \cdot \operatorname{Tr} E_{\lambda}^{\Lambda}(\beta)}{\operatorname{Tr} E_{\lambda}^{\Lambda}(\beta) \cdot \operatorname{Tr} E_{0}^{\Lambda}(\beta)} \\
\leqq \omega_{\lambda}^{\Lambda}(A) \frac{\left|\operatorname{Tr} E_{0}^{\Lambda}(\beta)-\operatorname{Tr} E_{\lambda}^{\Lambda}(\beta)\right|}{\operatorname{Tr} E_{0}^{\Lambda}(\beta)}+\frac{\left|\operatorname{Tr}\left(E_{\lambda}^{\Lambda}(\beta)-E_{0}^{\Lambda}(\beta)\right) A\right|}{\operatorname{Tr} E_{0}^{\Lambda}(\beta)},
\end{gathered}
$$

and

$\left|\omega_{\lambda}^{\Lambda}(A)-\omega_{0}^{\Lambda}(A)\right| \leqq \frac{2\|A\|}{\left\|E_{0}^{\Lambda}(\beta)\right\|_{1}}\left\|E_{\lambda}^{\Lambda}(\beta)-E_{0}^{\Lambda}(\beta)\right\|_{1}<|\lambda| \mathscr{C}_{\Lambda}, \mathscr{C}_{\Lambda}=\mathrm{const}$,

according to (17). Therefore, $\omega_{\lambda}^{\Lambda}$ is continuous at $\lambda=0$. Taking $E_{\lambda_{0}}^{\Lambda}(\xi)$, with arbitrary but fixed $\lambda_{0}$, instead of $E_{0}^{\Lambda}(\xi)$ as unperturbed semigroup, and introducing a small perturbation $\lambda W$, we can repeat the above arguments and thus get continuity of $\omega_{\lambda}^{\Lambda}$ at $\lambda_{0}$. Q.E.D.

Unfortunately, the constant $\mathscr{C}_{\Lambda}=4 c \xi\|A\| \frac{e^{\gamma_{\Lambda} \beta / 2}\left\|E_{0}^{\Lambda}(\beta / 2)\right\|_{1}}{\left\|E_{0}^{\Lambda}(\beta)\right\|_{1}}$ in is not independent of $\Lambda$, and it seems to be no easy task to prove uniform continuity.

4. In our simple model the states of the quantum systems are changed by the thermal coupling even in the case of equal temperatures. We assert that for $\beta_{1}=\beta_{2}$ in the limit $\Lambda_{i} \rightarrow \infty$ the partition functions remain the same, and in this sense the states are unchanged. Our arguments are necessarily indirect ones, and we do not claim to give an exact proof.

We let $\Lambda_{i}, i=1,2$, increase in such a way that $\Lambda_{1} \cap \Lambda_{2}=\emptyset$. Let us consider one of the systems and omit the index. Following Ruelle ([11], p. 65), we define the quantum microcanonical partition function

$$
\Omega(\Lambda, n, E)=\operatorname{Tr} \delta^{-}\left(H_{n}(\Lambda)-E\right)
$$


where

$$
\delta^{-}(x)=\left\{\begin{array}{lll}
1 & \text { if } & x<0 \\
0 & \text { if } & x>0
\end{array}\right.
$$

$H_{n}(\Lambda)$ is the $n$-particle Hamiltonian of the unperturbed system. Let $\varepsilon_{m}^{A}$ and $\varepsilon_{m}^{\Lambda}(\lambda)$ be the eigenvalues of $H_{n}(\Lambda)$ and $H_{n}(\Lambda)+\lambda W$, respectively. Because of $\|W\| \leqq c$, we again have for $\lambda>0$

$$
\varepsilon_{m}^{A}-\lambda c \leqq \varepsilon_{m}^{A}(\lambda) \leqq \varepsilon_{m}^{A}+\lambda c .
$$

$\Omega$ equals the number of eigenvalues of $H_{n}(\Lambda)$ below $E$; hence

$$
\begin{aligned}
\Omega(\Lambda, n, E-\lambda c) & \leqq \Omega_{\lambda}(\Lambda, n, E)=\operatorname{Tr} \delta^{-}\left(H_{n}(\Lambda)+\lambda W-E\right) \\
& \leqq \Omega(\Lambda, n, E+\lambda c) .
\end{aligned}
$$

The entropy of the system is given by $S(\Lambda, n, E)=\log \Omega(\Lambda, n, E)$ and $S_{\lambda}(\Lambda, n, E)=\log \Omega_{\lambda}(\Lambda, n, E)$, respectively. Eq. (19) implies

$$
S(\Lambda, n, E-\lambda c) \leqq S_{\lambda}(\Lambda, n, E) \leqq S(\Lambda, n, E+\lambda c) .
$$

For our discussion it is natural to require that the limit

$$
\lim \frac{1}{V} S(\Lambda)=s(\varrho, \varepsilon)
$$

exists when $\frac{E}{V} \rightarrow \varepsilon, \frac{n}{V} \rightarrow \varrho, V$ denoting the volume of $\Lambda$.

Since $\lambda c$ is fixed, it is clear that we can replace $E$ by $E+\lambda c$ without changing the result of the limiting procedure; thus we conclude from (20) that

$$
s(\varrho, \varepsilon)=s_{\lambda}(\varrho, \varepsilon)=\lim \frac{1}{V} S_{\lambda}(\Lambda)
$$

For the rest of the argument we again refer to Ruelle [11], where it is shown that different ensembles in the limit $\Lambda \rightarrow \infty$ yield the same physical quantities.

As we have seen, it is possible to introduce thermal interactions of certain infinite systems $S_{1}, S_{2}$ by considering the thermodynamic limit of finite systems with suitable interaction. Our model is very simple but it supports the conjecture that even for systems in thermal contact certain assumptions can be fulfilled which allow us to establish the existence of a common K.M.S. extension of two K.M.S. states of the respective systems.

Acknowledgement. I am indebted to Professor Borchers for stimulating discussions and to Professor Winnink for critical remarks, especially those concerning the strong continuity of the time automorphisms. 


\section{References}

1. Emch, G. G., Knops, H.J.F., Verboven, E.J.: J. Math. Phys. 11, 1655 (1970). A list of earlier references can be found there.

2. - - J. Math. Phys. 11, 3008 (1970).

3. Takesaki, M.: Commun. math. Phys. 17, 33 (1970).

4. Brascamp, H. J.: Commun. math. Phys. 18, 82 (1970).

5. Sirugue, M., Winnink, M.: Commun. math. Phys. 19, 161 (1970).

6. Lanford, O.E.: The KMS states of a quantum spin system, in: Systèmes à un nombre infini de degrés de liberté. Colloques internationeaux Gif-sur-Yvette 1969.

7. Haag, R., Hugenholtz, N. M., Winnink, M.: Commun. math. Phys. 5, 215 (1967).

8. Takesaki, M., Winnink, M.: Private communication by M. Winnink, to be published.

9. Slawny, J.: Tel Aviv University preprint TAUP-227-71.

10. Manuceau,J., Sirugue, M., Testard,D., Verbeure,A.: Marseille preprint 71/P. 397.

11. Ruelle, D. : Statistical Mechanics. New York: W. A. Benjamin, Inc. 1969.

12. Roos, H.: Commun. math. Phys. 16, 238 (1970).

13. Turumaru, T.: Tôhoku Math. J. 4, 242 (1952).

Takesaki, M.: Tôhoku Math. J. 16, 111 (1964).

14. Kato, T.: Perturbation theory for linear operators. Berlin-Heidelberg-New York: Springer 1966.

15. Robinson, D. W.: Commun. math. Phys. 7, 337 (1968).

H. Roos

Institut für Theoretische Physik

der Universität

D-3400 Göttingen, Bunsenstr. 9

Germany 